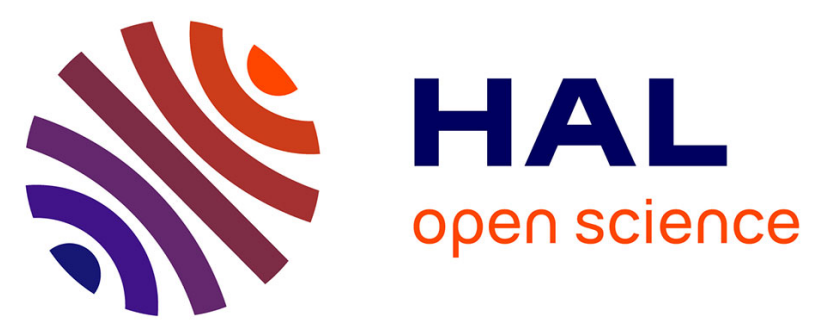

\title{
ÉTUDE ANALYTIQUE ET NUMÉRIQUE DU COMPORTEMENT EN DÉTENTE D'UN MATÉRIAU POREUX APRÈS UN CHOC DE COURTE DURÉE
}

\author{
A. Migault, D. Zagouri, J. Romain
}

\section{- To cite this version:}

A. Migault, D. Zagouri, J. Romain. ÉTUDE ANALYTIQUE ET NUMÉRIQUE DU COMPORTEMENT EN DÉTENTE D'UN MATÉRIAU POREUX APRÈS UN CHOC DE COURTE DURÉE. Journal de Physique IV Proceedings, 1991, 01 (C3), pp.C3-867-C3-872. 10.1051/jp4:19913121 . jpa00249926

\section{HAL Id: jpa-00249926 https://hal.science/jpa-00249926}

Submitted on 1 Jan 1991

HAL is a multi-disciplinary open access archive for the deposit and dissemination of scientific research documents, whether they are published or not. The documents may come from teaching and research institutions in France or abroad, or from public or private research centers.
L'archive ouverte pluridisciplinaire HAL, est destinée au dépôt et à la diffusion de documents scientifiques de niveau recherche, publiés ou non, émanant des établissements d'enseignement et de recherche français ou étrangers, des laboratoires publics ou privés. 


\title{
ÉTUDE ANALYTIQUE ET NUMERIQUE DU COMPORTEMENT EN DÉTENTE D'UN MATÉRIAU POREUX APRÈS UN CHOC DE COURTE DUREFE
}

\author{
A. MIGAULT, D. ZAGOURI et J.P. ROMAIN \\ L.E.D.-U.A.193-C.N.R.S, E.N.S.M.A, F-86034 Poitiers, France
}

Résumé - Cette étude présente une analyse du comportement des matériaux poreux en détente, derrière un front de choc. La vitesse matérielle résiduelle à pression nulle dépend de la pression de choc. On montre qu'elle passe par un maximum dont la valeur est déterminée en fonction de la porosité initiale du milieu, pour l'aluminium et pour le cuivre.

Abstract: In this paper, we present some results about the release waves in porous materials ( $\mathrm{Al}$ and $\mathrm{Cu}$ ) from any shocked state and for porosity between 1 (compact) and $\mathrm{L}_{\mathrm{lim}}\left(=1+2 / \gamma_{0} ; \gamma_{0}\right.$, Gruneisen coefficient of compact material. We describe the evolution of residual material velocity with the pressure of shocked state. This velocity has a maximum value which is positive; we give the evolution of this maximum with porosity for $\mathrm{Al}$ and $\mathrm{Cu}$.

\section{I - INTRODUCTION}

Lorsqu'une onde de choc passe d'un milieu dense, $\mathrm{A}$, à un milieu $\mathrm{B}$, tel que l'air, l'onde se réfléchie à l'interface et repart vers $A$. L'onde réfléchie dans le milieu $A$ est un faisceau de détente qui va diminuer progressivement la pression dans le milieu A et propager un accroissement de vitesse particulaire en sens inverse de sa propre propagation. Les lois gouvernant de tels écoulements sont celles des écoulements isentropiques $/ 1 /$.

Dans le plan $\left(\mathrm{P}, \mathrm{u}_{\mathrm{p}}\right)$ ( up ,vitesse particulaire dans le choc),la situation est la suivante:

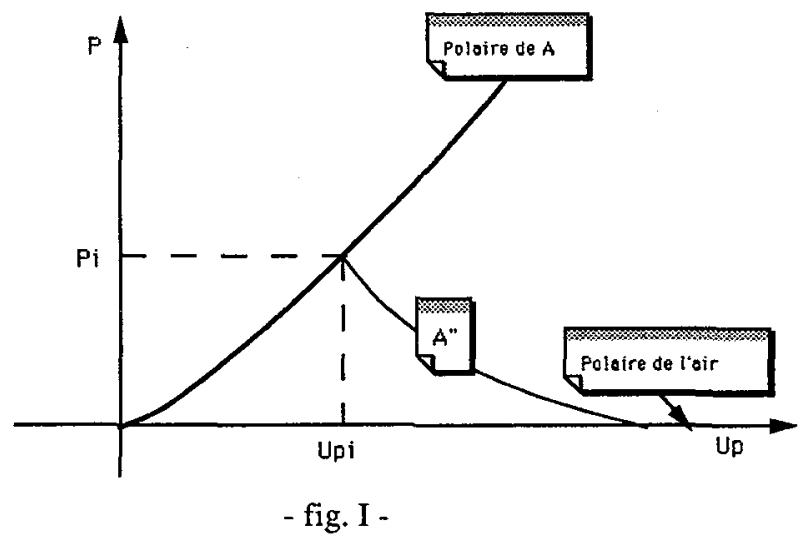


Le lieu des nouveaux états établis dans le milieu A est la courbe A" de la figure I; il est tel que

$$
u_{p}=u_{p i}=\int_{P_{i}}^{P}\left(-\frac{d V}{d P S}\right)^{1 / 2} d P
$$

$\mathrm{P}_{\mathrm{S}}(\mathrm{V})$ est la courbe de détente isentropique à partir de l'état de choc $\left(\mathrm{P}_{\mathbf{i}}, \mathrm{V}_{\mathrm{i}}, \mathrm{u}_{\mathrm{pi}}\right)$.

L'étude des corps poreux a été entreprise dans les années 60 pour obtenir des données sur l'équation d'état des solides à grande énergie interne et haute température $/ 2 /$. Ces corps se sont également révélés être de puissants amortisseurs de choc, $/ 3 /$; c'est la raison pour laquelle nous avons entrepris l'étude numérique des ondes de détente đans le cas d'un modèle simple de poreux.

\section{II - MODELE MATHEMATIQUE UTILISE}

Nous allons examiner plus particulièrement le problème dans le cas des corps poreux à "compaction complète". Dans ce cas, la courbe de Hugoniot du poreux, $\mathrm{P}_{\mathrm{H}}^{*}(\mathrm{~V})$, se déduit de la courbe de Hugoniot du compact, $\mathrm{P}_{\mathrm{H}}(\mathrm{V})$, à l'aide de $(/ 4 /, / 5 /)$ :

$$
P_{H}^{*}(V)=P_{H}(V) \frac{1-\frac{\gamma_{0}}{2 V_{0}}\left(V_{0}-V\right)}{1-\frac{\gamma_{0}}{2 V_{0}}\left(V_{0}^{*}-V\right)}
$$

$\mathrm{V}_{\mathrm{o}}^{*}$ est le volume spécifique initial du corps poreux à $\mathrm{P}=0$ et $\mathrm{T}=\mathrm{T}_{0} ; \mathrm{V}_{0}$ est le volume spécifique initial du corps compact (dans les mêmes conđitions).

Dans ce qui suit $\mathrm{L}$ désigne la porosité du milieu $; \mathrm{L}=\mathrm{V}_{\mathrm{o}}^{*} / \mathrm{V}_{\mathrm{o}}$.

La relation (2) est obtenue en supposant que le compact et le poreux ont une équation d'état du type MIE-GRUNEISEN /6/, que le rapport $\gamma(\mathrm{V}) / \mathrm{V}$ est constant et égal à $\gamma_{0} / \mathrm{V}_{0}$ ( $\gamma_{0}$, cofficient de Gruneisen et $V_{0}$, volume spécifique initial du corps compact) et que les équations de conservation de l'énergie, de la matière et de l'impulsion dans un choc sont satisfaites.

A partir d'un état de choc $\left(\mathrm{P}_{\mathrm{i}}^{*}, \mathrm{~V}_{\mathrm{i}}\right)$ le corps se détend de façon adiabatique. Compte tenu de (2) et des hypothèses faites pour y aboutir, on obtient l'équation différentielle suivante, donnant la loi d'évolution de la courbe de détente, $\mathrm{P}_{\mathrm{H}}^{*}(\mathrm{~V})$, connaissant la courbe de Hugoniot, $\mathrm{P}_{\mathrm{H}}^{*}(\mathrm{~V})$ :

$$
\left\{\begin{array}{c}
\frac{\mathrm{dP}_{\mathrm{S}}^{*}}{\mathrm{dV}}+\frac{\gamma_{\mathrm{o}}}{\mathrm{V}_{\mathrm{o}}} \mathrm{P}_{\mathrm{S}}^{*}=\frac{\gamma_{\mathrm{O}}}{2 \mathrm{~V}_{\mathrm{o}}} \mathrm{P}_{\mathrm{H}}^{*}+\frac{\mathrm{dP}_{\mathrm{H}}^{*}}{\mathrm{dV}}\left[1-\frac{\gamma_{\mathrm{o}}}{2 \mathrm{~V}_{\mathrm{o}}}\left(\mathrm{V}_{\mathrm{o}}^{*}-\mathrm{V}\right)\right] \\
\mathrm{V}=\mathrm{V}_{\mathrm{i}}, \quad \mathrm{P}_{\mathrm{S}}^{*}\left(\mathrm{~V}_{\mathrm{i}}\right)=\mathrm{P}_{\mathrm{H}}^{*}\left(\mathrm{~V}_{\mathrm{i}}\right) \equiv \mathrm{P}_{\mathrm{i}}^{*}
\end{array}\right.
$$

La polaire de détente, $\mathrm{P}_{\mathrm{H}}^{*}\left(\mathrm{U}_{\mathrm{p}}\right)$, s'obtient à partir de (3) et de la relation (1)

La courbe $\mathrm{P}_{S}^{*}\left(\mathrm{u}_{\mathrm{p}}\right)$ ainsi obtenue est la symétrique de $\mathrm{A}^{\prime \prime}$ par rapport à la droite $\mathrm{u}_{\mathrm{p}}=\mathrm{u}_{\mathrm{pi}}(\mathrm{fig} . \mathrm{I})$. 


\section{III - RESULTATS NUMERIQUES}

L'équation (2) montre l'existence d'une valeur de la porosité, $\mathrm{L}_{\mathrm{lim}}$ :

$\mathrm{L}_{\mathrm{lim}}=\frac{\left(\mathrm{V}_{\mathrm{o}}^{*}\right)_{\mathrm{lim}}}{\mathrm{V}_{\mathrm{o}}}=1+\frac{2}{\gamma_{\mathrm{o}}}$

Pour cette valeur de $\mathrm{L}$, la courbe de Hugoniot du poreux $\mathrm{P}_{\mathrm{H}}^{*}(\mathrm{~V})$, est une droite verticale parallèle à l'axe des pressions en $\mathrm{V}=\mathrm{V}_{\mathrm{O}}$. Cette remarque justifie le choix des corps pris pour les applications numériques. Nous avons pris l'aluminium 2024 et le cuivre qui, ayant même valeur de $\gamma_{0}$, ont même porosité limite, $\mathrm{L}_{\mathrm{lim}}=2$. Ces corps ont été examinés en détente pour différentes pressions initiales $\left(\mathrm{P}_{\mathrm{i}}^{*}\right)$ et différentes valeurs de la porosité $\left(\mathrm{L}=\mathrm{V}_{\mathrm{O}}^{*} / \mathrm{V}_{\mathrm{O}}\right)$. Qualitativement, les résultats obtenus sont les mêmes dans les deux cas.

\section{III-1 - Courbes de détente dans le plan $\left(P, u_{p}\right)$}

La figure II montre, dans le plan ( $\mathrm{P}, \mathrm{u}_{\mathrm{p}}$ ) la position respective de la polaire de choc du poreux $\left[\mathrm{P}_{\mathrm{H}}^{*}\left(\mathrm{u}_{\mathrm{p}}\right)\right]$ et de diverses polaires de détente $\left[\mathrm{P}_{\mathrm{S}}^{*}\left(\mathrm{u}_{\mathrm{p}}\right)\right]$ issues de différents états initiaux $\left(\mathrm{P}_{\mathrm{i}}{ }^{*}, \mathrm{u}_{\mathrm{pi}}\right)$.

L'examen de cette figure fait apparaître trois domaines de pression :

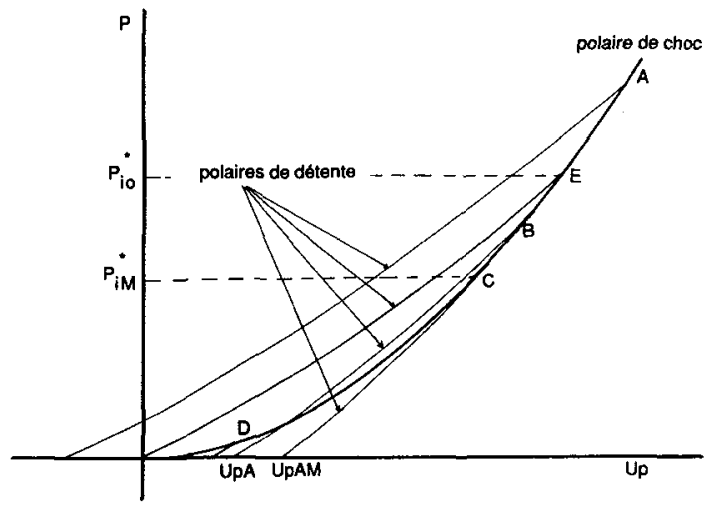

-fig.II-

- Si $\mathbf{P}_{i}^{*}$ est supérieure à $P_{i o}^{*}$, la polaire de détente, $P_{S}^{*}\left(u_{p}\right)$, est entièrement au-dessus de la polaire de choc et la vitesse particulaire résiduelle, après retour à la pression nulle, $u_{p} A$, est négative C'est un comportement analogue à celui d'un corps compact.

- Si $\mathrm{P}_{\mathrm{i}}^{*}=\mathrm{P}_{\text {io }}^{*}$, le corps revient au repos complet $; \mathrm{P}_{\mathrm{S}}^{*}=0$ et $\mathrm{upA}=0$.

- Si $\mathrm{P}_{\mathrm{i}}^{*}$ continue à décroître en dessous de $\mathrm{P}_{\text {io }}^{*}$, la courbe de détente recoupe la polaire de choc et la vitesse particulaire résiduelle, $u_{p} A$, est positive. Cette vitesse augmente jusqu'à une valeur $u_{p} A M$ 
atteinte pour une valeur $\mathrm{P}_{\mathrm{i}}^{*}=\mathrm{P}_{\mathrm{iM}}^{*}$. En ce point, courbe de détente, $\mathrm{P}_{\mathrm{S}}^{*}\left(\mathrm{u}_{\mathrm{p}}\right)$, et polaire de choc $\mathrm{P}_{\mathrm{H}}^{*}\left(\mathrm{u}_{\mathrm{p}}\right)$, sont tangentes.

- $\mathrm{Si} \mathrm{P}_{\mathrm{i}}^{*}$ continue à décroître en dessous de $\mathrm{P}_{\mathrm{iM}}^{*}$, la polaire de détente est entièrement au-dessous de la polaire de choc de poreux. La vitesse particulaire résiduelle $u_{p} A$ est toujours positive mais décroît jusqu'à 0 en même temps que $\mathbf{P}_{\mathbf{i}}^{*}$.

La figure III montre la courbe décrivant les variations de $u_{p} A$ en fonction de la pression initiale, $P_{i}^{*}$ dans le cas de l'aluminium de porosité $L=1,4$. Les points marqués $A, B, C, D$ et $E$ correspondent aux points $\mathrm{A}, \mathrm{B}, \mathrm{C}, \mathrm{D}$ et $\mathrm{E}$ de la figure II. Nous noterons que cette courbe a une tangente verticale à l'origine. Sur cette courbe, nous avons fait figurer les variations de la vitesse particulaire résiduelle en fonction de la pression initiale pour un corps compact : cette vitesse est toujours négative.

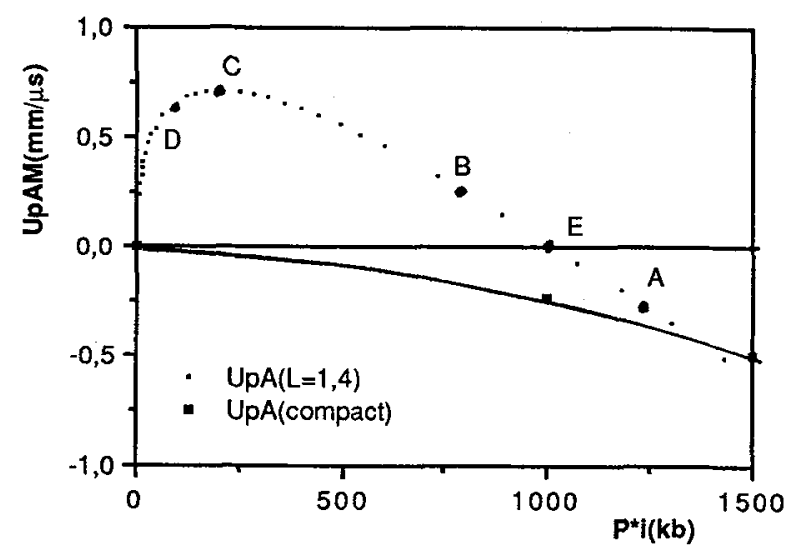

-fig III-

\section{III-2 - Courbes de détente dans le plan (P;V)}

Pour une porosité donnée, les courbes de détente, $\mathrm{P}_{\mathrm{S}}^{*}(\mathrm{~V})$, se placent régulièrement les unes audessus des autres à pression initiale $\mathrm{P}_{\mathrm{i}}$ croissante. Le volume résiduel, $\mathrm{V}_{\mathrm{A}}$, après retour à la pression nulle, est une fonction monotone croissante de $\mathrm{P}_{\mathrm{i} \text {. }}^{*}$ Pour $\mathrm{P}_{\mathrm{i}}^{*}=\mathrm{P}_{\mathrm{io}}^{*}$, on a $\mathrm{V}_{\mathrm{A}}=\mathrm{V}_{\mathrm{O}}^{*}$; on a également $(\S \mathrm{II}-1), \mathrm{u}_{\mathrm{pA}}=0 ;$ le corps est revenu au repos complet.

\section{III-3 - Résultats numériques}

Les résultats obtenus dans le cas du cuivre et de l'aluminium sont directement comparables car ils ont la même porosité limite $\left(\mathrm{L}_{\mathrm{lim}}=2\right)$ ). Les figures IV et $\mathrm{V}$ montrent l'évolution de $\mathrm{u}_{\mathrm{p}} \mathrm{AM}$ (vitesse résiduelle maximum) et de $\mathrm{P}_{\text {io }}^{*}$ (pression initiale de début de détente pour laquelle la vitesse résiduelle 
$u_{p} A$, est nulle) en fonction de la porosité. On constate que $u_{p} A M$, est une fonction croissante de $L$ et que les valeurs obtenues pour $\mathrm{Al}$ sont systématiquement au-dessus de celles obtenues pour $\mathrm{Cu}$.(cf.fig IV)

Les variations de $P_{i o}$ avec $L$ sont plus complexes mais ont les mêmes caractéristiques pour ces deux corps. Les valeurs obtenues pour le cuivre sont toujours supérieures à celles obtenues dans l'aluminium (voir fig. V).

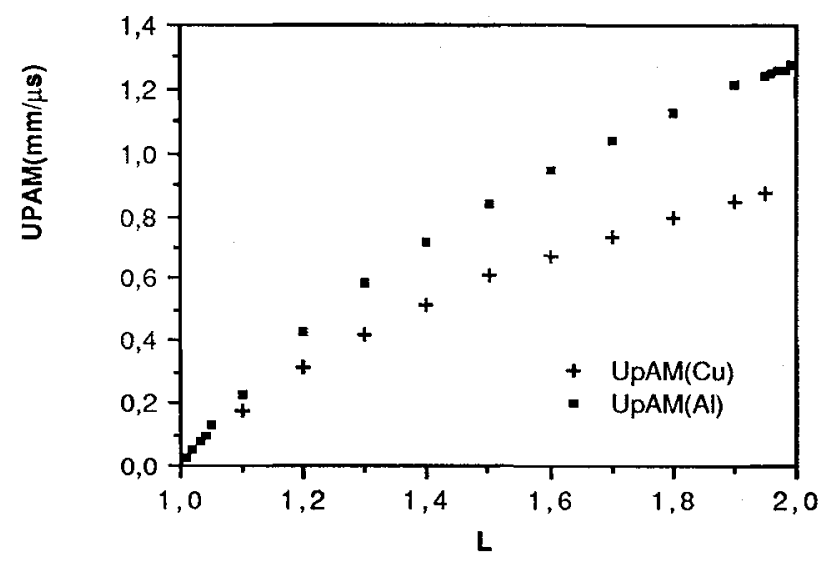

-fig. IV-

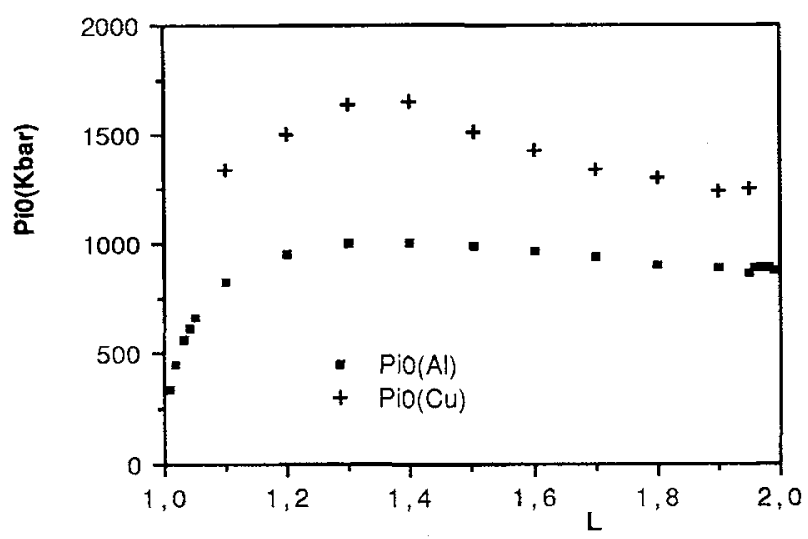

-fig V-

Le plomb et le polyurethane ont des porosités limites très différentes et les résultats numériques sont difficilement comparables entre eux et avec ceux du cuivre et de l'aluminium car nous n'avons pas réussi à mettre en évidence des relations d'échelle permettant une comparaison effective. Toutefois, la description qualitative présentée ci-dessus s'applique également à ces matériaux. 


\section{IV - INTERPRETATION DES RESULTATS ET CONCLUSION}

Géométriquement, dans le plan ( $\mathrm{P}, \mathrm{up})$ : il est aisé de montrer, si on assimile les courbes de détente avec des droites parallèles entre elles, que des variations comme celles montrées sur la figure III résultent uniquement de la courbure des polaires de choc du poreux qui sont de la forme up $\mathrm{p}^{\mathrm{b}}$ où $b$ est compris entre 1 (pour L=1) et 2 (pour L=Llim)

Le passage des valeurs négatives à des valeurs positives et l'existence d'un maximum de la vitesse particulaire résiduelle, upA, peut s'interpréter par la compétition entre deux effets opposés : réduction de volume associée au compactage et dilatation liée à l'énergie résiduelle qui augmente avec la pression. L'effet de la dilatation domine l'effet de compactage lorsque la température résiduelle dépasse un seuil déterminé par $\mathrm{P}_{\text {io }}^{*}$. On trouve une situation analogue dans le comportement d'un gaz parfait contenu dans un cylindre fermé par un piston mobile, à l'équilibre à la pression $P_{0}$ et au volume $V_{0}: s i$ on laisse tomber sur le piston une masse d'une hauteur suffisamment grande, la position finale du piston est au-dessus de sa position initiale ; l'augmentation de température due à la compression adiabatique irréversible produit une augmentation de volume supérieure à la diminution de volume produit par l'augmentation de pression.

Cette situation va entraîner, pour certaines conditions initiales, l'existence d'une série de choc et de détente secondaire dans le matériau. Cette possibilité a été analysée par ROMAIN et ZAGOURI /6/ mais, dans l'immédiat, sa mise en évidence expérimentale se heurte à de nombreuses difficultés

\section{BIBLIOGRAPHIE}

/1/ M.H. RICE and all. - Compression of solids by strong shock waves, Sol. St. Physics, 6 (1958) 1.

12/ Ya. B. ZEL'DOVICH et Yu. P. RAIZER - Physics of shock waves and High-temperature hydrodynamic phenomena, Academic Press - 1967.

13/ M.DEFOURNEAUX-Sciences et Techniques de l'Armement - Mémorial de l'Artillerie Française - 46 (1972) 695

14/ R.G. Mc QUEEN and Coll. - In "High velocity Impact phenomena" Ed. R. KINSLOW Academic Press - New York - 1970 - p. 294.

15/ K.I. KONDO - "In high pressure explosive processings of ceramics", Ed. R.A. GRAHAM and A.B. SAWAOKA - Trans. Techn. Pub. (Suisse) 1987 - p. 229.

/6/ J.P. ROMAIN et D. ZAGOURI - In" shock waves and high strain rates", Ed. M.A. MEYERS, L.E. MURR and K.P. STANDHAMMER, M. Dekker, New York, 1991. 